\title{
Effects of emission from different UWB short-range communication devices
}

\begin{abstract}
Ultra-wideband (UWB) technology is a new concept in wireless short-range communication. It has numerous advantages that are resulted in producing different new products in various applications. This technology uses very short duration pulses hence occupies very large transmission bandwidth. This phenomenon may cause harmful interference in the existing wireless devices. The main objective of this study is to evaluate the effective parameters in ultra-wideband communication for indoor short-range devices. In this regard, two commercial UWB devices are utilized and effects of these devices in different experimental configurations are investigated. Three configurations including the effects of distance, narrowband spectrum, and another UWB device on bit error rate performance are evaluated. The results of the performed experiments showed the stability and amenability of the UWB technology to be used in multiple UWB devices.
\end{abstract}

Keyword: Ultra-wideband; Emission; Interference; Short-range communication 